\title{
Therapeutic Hypothermia Reduces Oxidative Damage and Alters Antioxidant Defenses after Cardiac Arrest
}

\author{
Fernanda S. Hackenhaar, ${ }^{1,2}$ Tássia M. Medeiros,, ${ }^{1,2}$ Fernanda M. Heemann,, \\ Camile S. Behling, ${ }^{1,2}$ Jordana S. Putti, ${ }^{1,2}$ Camila D. Mahl, ${ }^{1,2}$ Cleber Verona, ${ }^{1,2,3}$ \\ Ana Carolina A. da Silva, ${ }^{1,2}$ Maria C. Guerra, ${ }^{4}$ Carlos A. S. Gonçalves, ${ }^{4}$ Vanessa M. Oliveira, ${ }^{3}$ \\ Diego F. M. Riveiro, ${ }^{3}$ Silvia R. R. Vieira, ${ }^{5}$ and Mara S. Benfato ${ }^{1,2}$ \\ ${ }^{1}$ Departamento de Biofísica, UFRGS, Porto Alegre, Brazil \\ ${ }^{2}$ Programa de Pós-Graduação em Biologia Celular e Molecular, UFRGS, Porto Alegre, Brazil \\ ${ }^{3}$ Grupo Hospitalar Conceição, Porto Alegre, Brazil \\ ${ }^{4}$ Instituto de Ciências Básicas da Saúde, UFRGS, Porto Alegre, Brazil \\ ${ }^{5}$ Hospital de Clínicas de Porto Alegre, Porto Alegre, Brazil
}

Correspondence should be addressed to Mara S. Benfato; mara.benfato@ufrgs.br

Received 22 December 2016; Revised 7 February 2017; Accepted 21 February 2017; Published 2 May 2017

Academic Editor: Silvana Hrelia

Copyright (C) 2017 Fernanda S. Hackenhaar et al. This is an open access article distributed under the Creative Commons Attribution License, which permits unrestricted use, distribution, and reproduction in any medium, provided the original work is properly cited.

\begin{abstract}
After cardiac arrest, organ damage consequent to ischemia-reperfusion has been attributed to oxidative stress. Mild therapeutic hypothermia has been applied to reduce this damage, and it may reduce oxidative damage as well. This study aimed to compare oxidative damage and antioxidant defenses in patients treated with controlled normothermia versus mild therapeutic hypothermia during postcardiac arrest syndrome. The sample consisted of 31 patients under controlled normothermia $\left(36^{\circ} \mathrm{C}\right)$ and 11 patients treated with $24 \mathrm{~h}$ mild therapeutic hypothermia $\left(33^{\circ} \mathrm{C}\right)$, victims of inor out-of-hospital cardiac arrest. Parameters were assessed at 6, 12, 36, and $72 \mathrm{~h}$ after cardiac arrest in the central venous blood samples. Hypothermic and normothermic patients had similar S100B levels, a biomarker of brain injury. Xanthine oxidase activity is similar between hypothermic and normothermic patients; however, it decreases posthypothermia treatment. Xanthine oxidase activity is positively correlated with lactate and S100B and inversely correlated with $\mathrm{pH}$, calcium, and sodium levels. Hypothermia reduces malondialdehyde and protein carbonyl levels, markers of oxidative damage. Concomitantly, hypothermia increases the activity of erythrocyte antioxidant enzymes superoxide dismutase, glutathione peroxidase, and glutathione S-transferase while decreasing the activity of serum paraoxonase-1. These findings suggest that mild therapeutic hypothermia reduces oxidative damage and alters antioxidant defenses in postcardiac arrest patients.
\end{abstract}

\section{Introduction}

Postcardiac arrest syndrome (PCAS) refers to the pathophysiological consequences of return of spontaneous circulation (ROSC) after successful cardiopulmonary resuscitation (CPR) for cardiac arrest (CA). PCAS comprises brain injury, hemodynamic dysfunction, damage to the heart and other organs, sepsis, and systemic inflammation consequent to ischemia-reperfusion (I/R) injury. The first 3 days after
CA are considered the critical phase of PCAS, when patients develop progressive organ damage and increased inflammation [1].

During PCAS, damage to the brain, heart, and other organs resulting from I/R has been attributed to oxidative stress, as the sudden return of oxygen to circulation increases the generation of reactive oxygen species (ROS) $[2,3]$. Generation of ROS, especially superoxide radical, increases dramatically after reperfusion and depends on xanthine 
oxidoreductase (XOR) and other pathways [4, 5]. Endothelial XOR plays a well-known role in free radical generation during hypoxia and reperfusion, when xanthine dehydrogenase $(\mathrm{XDH})$ is converted to xanthine oxidase $(\mathrm{XO})$, using oxygen as an electron acceptor instead of nicotinamide adenine dinucleotide $\left(\mathrm{NAD}^{+}\right)$, thus generating superoxide and hydrogen peroxide [6].

Post-CA hypoxia leads not only to oxidative stress but also to marked metabolic acidosis. Anaerobic glycolysis decreases the glucose concentration within the tissues, while increasing lactate formation. Concomitantly, a compensatory mechanism to restore $\mathrm{pH}$ increases the levels of calcium, sodium, and other electrolytes, such as potassium and magnesium [7]. Elevated blood lactate levels are found in patients with poor outcome after CA, since these levels are associated with the time to achieve ROSC [8].

Mild therapeutic hypothermia (MTH) has been the only clinical treatment applied to reduce post-CA injury, and clinical and animal model studies have supported the benefits of MTH for improving brain recovery [9-11]. MTH also prevents damage to other tissues, including the myocardium, liver, and kidney [12]. Damage protection by MTH is reinforced by studies demonstrating reduced oxidative damage after treatment [11-13]. Hypothermia may also be involved in preventing acidosis and lactate clearance, thereby restoring normal metabolism $[14,15]$.

The present study aimed to investigate oxidative stress during PCAS by assessing oxidative stress parameters at 6 , 12,36 , and $72 \mathrm{~h}$ after CA in intensive care unit (ICU) patients and by comparing patients treated with controlled normothermia versus MTH.

\section{Materials and Methods}

2.1. Screening of Patients. This prospective observational cohort study was conducted between May 2011 and October 2012 and included patients admitted to the ICU of Hospital Conceição, a public hospital located in Porto Alegre, Brazil. The hospital has 843 beds and 59 ICU beds and treats approximately 25,900 patients per year. The study was approved by the institutional ethics committee, and written informed consent was obtained from the relatives of all participants.

The study screened comatose patients, victims of inhospital or out-of-hospital CA, who had successful ROSC within $20 \mathrm{~min}$ of $\mathrm{CPR}$. Patients were assigned to receive either actively controlled normothermia $\left(36^{\circ} \mathrm{C}, n=31\right)$ or MTH $\left(33^{\circ} \mathrm{C}, n=11\right)$. Patients were assigned to MTH according to the availability of the room equipped for this purpose. All included patients had monitored temperature $\leq 37^{\circ} \mathrm{C}$, were in a state of coma (Glasgow Coma Scale score $\leq 8$ ), were receiving adequate oxygenation by mechanical ventilation, as indicated by $\mathrm{PaO}_{2} \geq 60$ torr $(\geq 8 \mathrm{kPa})$ with $\mathrm{FiO}_{2} \leq 0.4$ and positive end-expiratory pressure $\leq 8 \mathrm{~cm} \mathrm{H}_{2} \mathrm{O}$, and were cardiovascularly stable (heart rate $\leq 130$ beats/min) with controlled acid-base balance and electrolyte levels. Exclusion criteria were patients aged $<18$ years, trauma victims, postoperative patients ( $<7$ days after operation), patients who experienced more than one CA event during admission, pregnant women, and patients with advanced cancer. The full medical history of each patient was evaluated. Longterm survival was followed for 3 years after CA by telephone contact with the patient or family members.

2.2. Mild Therapeutic Hypothermia (MTH). Post-CA MTH was applied according to the hospital protocol. Cooling started $4 \mathrm{~h}$ after CA aiming at $32-34^{\circ} \mathrm{C}$. A combined cooling method was applied, including the use of endovascular cooling, ice packs, air conditioning, and thermal blanket for controlled cooling and rewarming of patients (circulating-water mattress; Medi-Therm ${ }^{\circledR}$ III Hyper/Hypothermia Machine MTA 6900, Gaymar Industries Inc.). Body temperature was measured continuously by an esophageal temperature probe. Neuromuscular-blocking agents (atracurium and/or pancuronium) were administered to control chills, thereby preventing heating of the body. Rewarming to the target temperature of $36^{\circ} \mathrm{C}$ started $24 \mathrm{~h}$ after hypothermia and did not exceed $0.25-0.5^{\circ} \mathrm{C} / \mathrm{h}$. Hyperthermia was promptly treated using antipyretic drugs in both normothermic and hypothermic patients, since temperature above $37^{\circ} \mathrm{C}$ is associated with poor outcome [16].

2.3. Clinical and Laboratory Data Assessment and Sample Collection. Clinical and laboratory data and venous blood samples were obtained from both normothermic and hypothermic patients at $6,12,36$, and $72 \mathrm{~h}$ after CA. Clinical data analysis included blood pressure, internal body temperature, heart rate, and respiratory rate. Laboratory data were assessed in venous blood by the hospital biochemistry staff and included $\mathrm{pH}$, glucose, lactate, and electrolyte levels. High-sensitivity C-reactive protein (hs-CRP), hematocrit, and hemoglobin levels were analyzed daily by the hospital biochemistry staff.

Venous blood was drawn from the central venous catheter for analysis of oxidative stress and neuronal injury biomarkers. A total of $10 \mathrm{~mL}$ of blood was prepared for isolation of serum, plasma (citrate), and erythrocytes. Samples were stored in liquid nitrogen within $30 \mathrm{~min}$ of blood collection and posteriorly stored at $-80^{\circ} \mathrm{C}$. Erythrocytes were lysed and diluted $1: 100$ in $2 \%$ ethanol and $10 \mu \mathrm{mol} / \mathrm{L}$ protease inhibitor phenylmethylsulfonyl fluoride.

\subsection{Neuronal Injury and Oxidative Stress Biomarkers}

2.4.1. Serum S100 Calcium-Binding Protein Beta (S100B) Levels. S100B levels were determined using an in-house ELISA method. Serum samples were coated with anti-S100B monoclonal antibody (SH-B1, Sigma-Aldrich) and then incubated with anti-S100 polyclonal antibody (DAKO), followed by incubation with peroxidase-conjugated anti-rabbit antibody (Sigma-Aldrich). Reaction with o-phenylenediamine was measured using a spectrophotometer at a wavelength of $492 \mathrm{~nm}$ [17].

2.4.2. Plasma Carbonyl Levels. Plasma was incubated with 2,4dinitrophenylhydrazine for analysis of carbonyl content using a spectrophotometer at $370 \mathrm{~nm}$. Hydrazine $\left(\varepsilon_{370}{ }^{\mathrm{M}}\right.$ $21,000 \mathrm{M}^{-1} \mathrm{~cm}^{-1}$ ) was used to determine carbonyl levels [18]. 
2.4.3. Serum Malondialdehyde (MDA) Levels. Serum MDA levels were measured by high-performance liquid chromatography at $250 \mathrm{~nm}$ (LC-18 DB column) [19].

2.4.4. Xanthine Oxidase (XO) Activity. Serum XO activity was measured using a XO fluorometric assay kit (Cayman Chemical, USA), applying an excitation wavelength of $520-550 \mathrm{~nm}$ and an emission wavelength of 585-595 nm.

2.4.5. Superoxide Dismutase (SOD) Activity. SOD activity was measured in erythrocyte lysate samples by spectrophotometry using the RanSOD kit (Randox, UK).

2.4.6. Glutathione Peroxidase (GPx) Activity. GPx activity was measured in erythrocyte lysate samples by spectrophotometry using the RanSel kit (Randox, UK).

2.4.7. Glutathione S-Transferase (GST) Activity. GST activity in erythrocyte lysate samples was determined by spectrophotometry by measuring the conjugation of 1-chloro-2,4-dinitrobenzene and glutathione forming S-(2,4-dinitrophenyl)glutathione $\left(\varepsilon_{340}{ }^{\mathrm{M}} 9600 \mathrm{M}^{-1} \mathrm{~cm}^{-1}\right)$ [20].

2.4.8. Paraoxonase-1 (PON1) Activity. Serum PON1 activity was measured by spectrophotometry at $412 \mathrm{~nm}$ using paraoxon as substrate, based on the formation of p-nitrophenol $\left(\varepsilon_{412}{ }^{\mathrm{M}} 169,000 \mathrm{M}^{-1} \mathrm{~cm}^{-1}\right)$ by paraoxon hydrolysis $[21,22]$.

2.4.9. Data Normalization. All assays were performed in triplicate. The results were normalized for total protein content by the Bradford method, using bovine serum albumin as standard [23].

2.5. Statistical Analysis. PASW Statistics 18 (SPPS Inc.) was used to construct multivariable logistic regression models with generalized estimating equations in order to compare the parameters of interest. Pairwise comparisons of estimated means by Bonferroni post hoc were used. Two-tailed Spearman's correlation was used to examine the relationship between variables, and the GNU Image Manipulation Program, version 2.8.16, was used to create the schematic representation of the correlations. Variables with normal distribution are presented as mean \pm standard error, and variables with nonnormal distribution are presented as median (min; max) or median and interquartile range. The sample size of 42 patients was calculated using the WINPEPI program. COMPARE2 was used to compare the difference of MDA mean \pm standard deviation between good and poor outcome groups from a previous study of cardiac arrest patients, to achieve $80 \%$ power at a $5 \%$ significance level [24].

\section{Results}

3.1. Baseline Characteristics and Outcome Biomarkers. Baseline characteristics of patients are shown in Table 1. Age and sex did not differ significantly between normothermic and hypothermic patients. ROSC also did not differ between groups, indicating that participants had similar baseline characteristics in both groups (Table 1).

A total of 42 patients were included in the study. Thirtyone patients received actively controlled normothermia $\left(36^{\circ} \mathrm{C}\right)$, while 11 patients received $\mathrm{MTH}\left(33^{\circ} \mathrm{C}\right)$. In hypothermic patients, body temperature reached $33^{\circ} \mathrm{C} 6 \mathrm{~h}$ after $\mathrm{CA}$, and this temperature was maintained for $24 \mathrm{~h}$ as observed at 6 and $12 \mathrm{~h}$ after CA. After $36 \mathrm{~h}$, body temperature reached $36^{\circ} \mathrm{C}$ (Table 2).

Blood hs-CRP levels, a biomarker of inflammation, were not significantly altered by hypothermia treatment. Other important biomarkers of outcome after CA were evaluated, including blood levels of calcium, sodium, glucose, and lactate. The levels of lactate, calcium, sodium, and $\mathrm{pH}$ were not altered in hypothermic patients. Blood lactate levels decreased significantly $12 \mathrm{~h}$ after CA in both groups and remained at the same levels at 36 and $72 \mathrm{~h}$. The decreased blood glucose levels found at the initial 6 and $12 \mathrm{~h}$ after CA were significantly increased in patients treated with hypothermia, returning to similar levels at 36 and $72 \mathrm{~h}$ after CA in both groups (Table 2).

3.2. Oxidative and Brain Damage Biomarkers. Serum S100B levels, a biomarker of brain injury, did not differ significantly in hypothermic patients. S100B levels decreased significantly $12 \mathrm{~h}$ after $\mathrm{CA}$ in both groups and remained at similar levels after 36 and $72 \mathrm{~h}$ (Figure 1 ).

MTH clearly reduced the oxidative damage parameters evaluated in the present study. Plasma carbonyl levels, a biomarker of protein oxidative damage, decreased significantly in patients treated with hypothermia at all time points after CA. Likewise, hypothermic patients had decreased serum MDA levels, a biomarker of lipid oxidative damage, at all time points after CA (Figure 1).

$\mathrm{XO}$ activity did not differ significantly between hypothermic and normothermic patients. However, in the hypothermic group, $\mathrm{XO}$ activity decreased at 36 and $72 \mathrm{~h}$ after CA, coinciding with the period of posthypothermia treatment (Figure 1).

3.2.1. Correlation Analysis. Due to its relevance to $\mathrm{I} / \mathrm{R}$ injury, XO activity was correlated with the biomarkers of outcome and acidosis and the results are summarized in Figure 2. XO activity was negatively correlated with venous $\mathrm{pH}(R=-0.360, p \leq 0.01)$ and positively correlated with blood lactate $(R=0.405, p \leq 0.001)$; venous $\mathrm{pH}$, in turn, was inversely correlated with blood lactate $(R=-0.336, p \leq 0.01)$. Conversely, XO activity was inversely correlated with blood calcium levels $(R=-0.543, p \leq 0.001)$ and blood sodium levels $(R=-0.332, p \leq 0.01)$. Importantly, serum S100B levels were positively correlated with XO activity $(R=0.412, p \leq 0.001)$ and blood lactate $(R=0.408$, $p \leq 0.001)$; conversely, serum S100B levels were inversely correlated with venous blood $\mathrm{pH}(R=-0.358, p \leq 0.01)$. No correlation was found between $\mathrm{XO}$ activity and MDA levels $(R=0.194, p=0.271)$ or carbonyl levels $(R=0.010, p=0.907)$.

3.3. Antioxidant Enzymatic Activities. The activities of antioxidant enzymes related to the detoxification of ROS were significantly altered by hypothermia treatment. The activities of erythrocyte antioxidant enzymes SOD, GPx, and GST increased significantly at $6,12,36$, and $72 \mathrm{~h}$ after 
TABLE 1: Baseline characteristics of 42 comatose in- and out-of-hospital cardiac arrest patients.

\begin{tabular}{|c|c|c|c|c|}
\hline & Normothermic $(n=31)$ & Hypothermic $(n=11)$ & All patients $(n=42)$ & $p$ value \\
\hline Age-years (mean \pm standard error) & $62.6 \pm 1.4$ & $60.6 \pm 1.6$ & $62.5 \pm 1.1$ & ns \\
\hline Male patients- $n(\%)$ & $20(64.5)$ & $7(63.6)$ & $27(64.3)$ & ns \\
\hline Time to ROSC $-\min (\operatorname{median} \pm \min ; \max )$ & $8 \pm(2 ; 20)$ & $6 \pm(4 ; 16)$ & $8 \pm(2 ; 20)$ & ns \\
\hline Survival—days (median \pm min; $\max$ ) & $8 \pm(1 ; 693)^{*}$ & $16 \pm(1 ; 163)$ & $8 \pm(1 ; 693)$ & ns \\
\hline \multicolumn{5}{|l|}{ Presumed cause of arrest-number } \\
\hline Presumed cardiac origin & $7 / 31$ & $3 / 11$ & $10 / 42$ & \\
\hline Presumed pulmonary origin & $6 / 31$ & $1 / 11$ & $7 / 42$ & \\
\hline Presumed hemorrhagic origin & $1 / 31$ & $3 / 11$ & $4 / 42$ & \\
\hline Presumed septic shock origin & $5 / 31$ & $4 / 11$ & $9 / 42$ & \\
\hline Unspecified origin & $12 / 31$ & & $12 / 42$ & \\
\hline \multicolumn{5}{|l|}{ Comorbidities_number } \\
\hline Acquired immunodeficiency syndrome & $5 / 31$ & $1 / 11$ & $6 / 42$ & \\
\hline Acute biliary pancreatitis & $1 / 31$ & & $1 / 42$ & \\
\hline Acute coronary syndrome & & $1 / 11$ & $1 / 42$ & \\
\hline Acute renal failure & & $1 / 11$ & $1 / 42$ & \\
\hline Acute respiratory infection & $2 / 31$ & & $2 / 42$ & \\
\hline Acute respiratory failure & & $1 / 11$ & $1 / 42$ & \\
\hline Anemia & & $1 / 11$ & $1 / 42$ & \\
\hline Anorexia & & $1 / 11$ & $1 / 42$ & \\
\hline Cancer & $2 / 31$ & $4 / 11$ & $6 / 42$ & \\
\hline Chronic obstructive pulmonary disease & $1 / 31$ & & $1 / 42$ & \\
\hline Cirrhosis & & $1 / 11$ & $1 / 42$ & \\
\hline Cytomegalovirus & $1 / 31$ & & $1 / 42$ & \\
\hline Chronic renal failure & $4 / 31$ & $1 / 11$ & $5 / 42$ & \\
\hline Congestive heart failure & $2 / 31$ & & $2 / 42$ & \\
\hline Diabetes mellitus & $2 / 31$ & $2 / 11$ & $4 / 42$ & \\
\hline Enterorrhagia & $1 / 31$ & $3 / 11$ & $4 / 42$ & \\
\hline Gastroenteritis & $2 / 31$ & $2 / 11$ & $4 / 42$ & \\
\hline Hypertension & $3 / 31$ & & $3 / 42$ & \\
\hline Hypothyroidism & $1 / 31$ & & $1 / 42$ & \\
\hline Iron deficiency anemia & $1 / 31$ & & $1 / 42$ & \\
\hline Ischemic heart disease & $3 / 31$ & $2 / 11$ & $5 / 42$ & \\
\hline Meningoencephalitis & $1 / 31$ & & $1 / 42$ & \\
\hline Myocardial infarction & $4 / 31$ & $1 / 11$ & $5 / 42$ & \\
\hline Peripheral vascular disease & $1 / 31$ & & $1 / 42$ & \\
\hline Pneumonia & $2 / 31$ & $1 / 11$ & $3 / 42$ & \\
\hline Pulmonary embolism & $2 / 31$ & & $2 / 42$ & \\
\hline Sepsis & $6 / 31$ & $4 / 11$ & $10 / 42$ & \\
\hline Stroke & $1 / 31$ & $1 / 11$ & $2 / 42$ & \\
\hline Tuberculosis & $2 / 31$ & $2 / 11$ & $4 / 42$ & \\
\hline Ulcer & $1 / 31$ & $1 / 11$ & $2 / 42$ & \\
\hline
\end{tabular}

ROSC: return of spontaneous circulation; * two patients in the normothermic group were alive 3 years after cardiac arrest.

CA in hypothermic patients, as compared to normothermic patients. Surprisingly, PON1 activity, an antioxidant enzyme present in high-density lipoproteins, showed an inverse profile as compared to all other antioxidant enzymes under analysis. Serum PON1 activity was significantly decreased in hypothermic patients at $6,12,36$, and $72 \mathrm{~h}$ after CA (Figure 3).
3.3.1. Correlation Analysis. The activities of erythrocyte antioxidant enzymes were strongly correlated. SOD activity was positively correlated with $\mathrm{GPx}(R=0.845, p \leq 0.001)$ and GST $(R=0.745, p \leq 0.001)$ activities. GPx was also strongly correlated with GST activity $(R=0.629, p \leq 0.001)$. Interestingly, serum PON1 activity did not correlate with the activities of the analyzed erythrocyte antioxidant 
TABLE 2: Biomarkers of outcome in patients with postcardiac arrest syndrome (PCAS).

\begin{tabular}{|c|c|c|c|c|c|c|c|c|}
\hline & \multicolumn{4}{|c|}{ Normothermic patients } & \multicolumn{4}{|c|}{ Hypothermic patients } \\
\hline & $6 \mathrm{~h}$ & $12 \mathrm{~h}$ & $36 \mathrm{~h}$ & $72 \mathrm{~h}$ & $6 \mathrm{~h}$ & $12 \mathrm{~h}$ & $36 \mathrm{~h}$ & $72 \mathrm{~h}$ \\
\hline Temperature, ${ }^{\circ} \mathrm{C}$ & $35.6 \pm 0.27$ & $35.7 \pm 0.21$ & $35.6 \pm 0.53$ & $36.1 \pm 0.15$ & $32.9 \pm 0.25^{+, \#}$ & $32.6 \pm 0.31^{+, \#}$ & $36.1 \pm 0.30$ & $36.1 \pm 0.20$ \\
\hline Hs-CRP, mg/L & $114.6 \pm 20.92$ & n.a. & $178.4 \pm 36.1$ & $141.2 \pm 44.3$ & $173.4 \pm 31.7$ & n.a. & $161.8 \pm 38.82$ & $205.9 \pm 65.18$ \\
\hline $\mathrm{S} 100 \mathrm{~B}, \mathrm{ng} / \mathrm{mL}$ & $\begin{array}{c}0.13 \\
(0.01 ; 6.63)^{+}\end{array}$ & $\begin{array}{c}0.07 \\
(0.01 ; 1.42)\end{array}$ & $\begin{array}{c}0.06 \\
(0.001 ; 1.8)\end{array}$ & $\begin{array}{c}0.50 \\
(0.01 ; 1.3)\end{array}$ & $\begin{array}{c}0.15 \\
(0.02 ; 1.8)^{+}\end{array}$ & $\begin{array}{c}0.09 \\
(0.04 ; 0.71)\end{array}$ & $\begin{array}{c}0.04 \\
(0.01 ; 0.6)\end{array}$ & $\begin{array}{c}0.03 \\
(0.02 ; 1.10)\end{array}$ \\
\hline Venous $\mathrm{pH}$ & $7.28 \pm 0.04$ & $7.36 \pm 0.04$ & $7.33 \pm 0.06$ & $7.35 \pm 0.03$ & $7.20 \pm 0.05$ & $7.28 \pm 0.05$ & $7.34 \pm 0.043$ & $7.32 \pm 0.07$ \\
\hline Glucose, mg/dL & $123.6 \pm 16.03^{+}$ & $126.6 \pm 8.47^{+}$ & $138.9 \pm 9.35$ & $167.3 \pm 24.11$ & $179.2 \pm 22.43^{+, \#}$ & $155.3 \pm 22.43^{\#}$ & $129.0 \pm 8.42$ & $107.3 \pm 11.22$ \\
\hline Lactate, $\mathrm{mmol} / \mathrm{L}$ & $2.9(0.8 ; 13.7)^{+}$ & $1.8(0.7 ; 17)$ & $1.4(0.7 ; 11.8)$ & $1.7(0.5 ; 8.5)$ & $1.9(0.6 ; 18)^{+}$ & $1.8(0.6 ; 19)$ & $1.6(1.0 ; 4.2)$ & $1.4(0.6 ; 1.5)$ \\
\hline Sodium, mmol/L & $136.4 \pm 1.54$ & $136.9 \pm 1.48$ & $136.2 \pm 1.70$ & $134.0 \pm 2.11$ & $135.5 \pm 2.46$ & $104.1 \pm 1.98$ & $134.6 \pm 3.36$ & $140.3 \pm 1.70$ \\
\hline Calcium, mg/dL & $1.04 \pm 0.025$ & $1.09 \pm 0.07$ & $1.05 \pm 0.04$ & $1.06 \pm 0.06$ & $1.05 \pm 0.02$ & $1.04 \pm 0.02$ & $1.03 \pm 0.03$ & $1.13 \pm 0.03$ \\
\hline
\end{tabular}

Hs-PCR: high-sensitivity C-reactive protein; data are expressed as mean \pm standard error, except for S100B and lactate, which are expressed as median (min; max); + is significantly different among time points within the normothermic or hypothermic group, $p \leq 0.05$; \# is significantly different when comparing normothermic versus hypothermic groups at the same time point, $p \leq 0.05$.

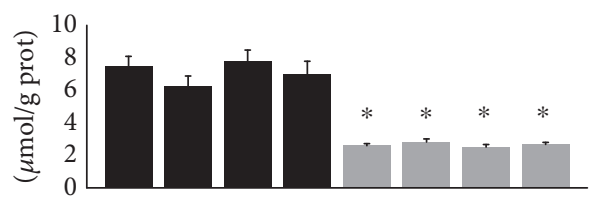

(a) Protein carbonyl levels

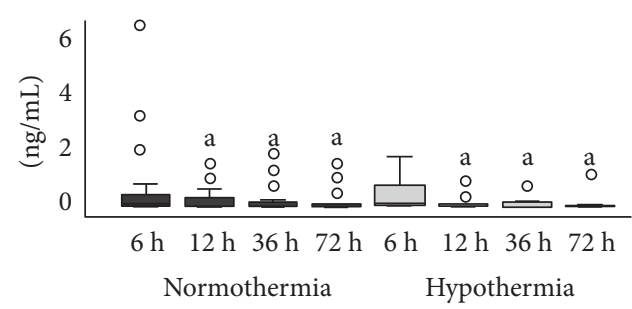

(c) S100B levels

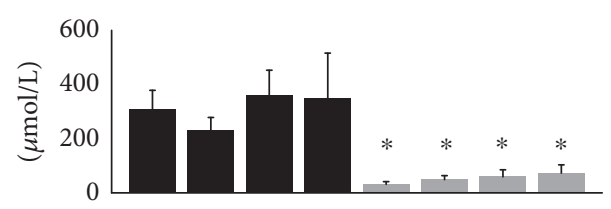

(b) Malondialdehyde levels

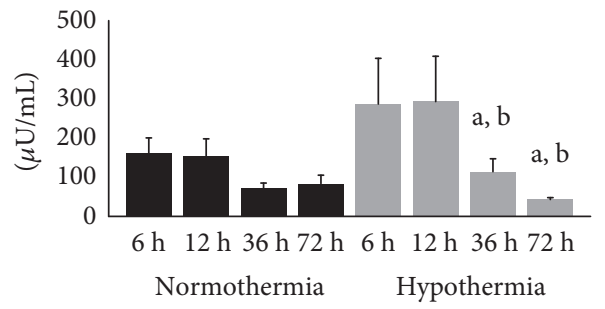

(d) Xanthine oxidase activity

Figure 1: Biomarkers of oxidative damage and brain injury at $6,12,36$, and $72 \mathrm{~h}$ after cardiac arrest $(\mathrm{CA})$ in normothermic $(n=31)$ versus hypothermic $(n=11)$ patients. In hypothermic patients, body temperature reached $33^{\circ} \mathrm{C}$ at 6 and $12 \mathrm{~h}$ and $36^{\circ} \mathrm{C}$ at 36 and $72 \mathrm{~h}$ after $\mathrm{CA}$. (a) Carbonyl levels, a biomarker of oxidative damage to proteins; (b) malondialdehyde levels, a biomarker of oxidative damage to lipids; (c) S100B levels, a biomarker of brain injury; (d) xanthine oxidase (XO) activity, a biomarker of generation of superoxide free radical. Data are expressed as mean \pm standard error, except for S100B levels, which are expressed as median and interquartile range. ${ }^{\circ}$ Significantly different when comparing normothermic versus hypothermic groups at the same time point, $p \leq 0.05$. ${ }^{\text {a Significantly }}$ different within the group when compared to $6 \mathrm{~h}, p<0.05$. ' Significantly different within the group when compared to $12 \mathrm{~h}, p \leq 0.05$.

enzymes (SOD, $R=-0.156, p=0.082 ; \mathrm{GPx}, R=-0.097$, $p=0.288$; GST, $R=-0.154, p=0.085$ ) (data not shown).

\section{Discussion}

The present study evaluated the effects of MTH on oxidative damage and antioxidant profile in post-CA patients. The results suggest that $\mathrm{MTH}$ reduces oxidative damage and increases the activity of most of the analyzed antioxidant enzymes. Indeed, carbonyl and MDA levels were significantly reduced while erythrocyte antioxidant enzymes SOD, GPx, and GST showed increased activity during PCAS in the hypothermic group, indicating that hypothermia reduces oxidative stress in post-CA patients.
4.1. Oxidative Damage and Xanthine Oxidase (XO) Activity. Oxidative stress has been implicated as a crucial factor in organ damage and hemodynamic dysfunction during PCAS, and, in this setting, MTH may be a suitable treatment option to reduce oxidative stress. $\mathrm{MTH}$ was found to decrease frontal cortex MDA and carbonyl levels $24 \mathrm{~h}$ after ROSC in a porcine model of CA [11].

In one of the few studies on oxidative stress in post-CA patients treated with hypothermia, plasma reactive oxygen metabolite (d-ROM) levels were decreased during the hypothermic stage of treatment $\left(33^{\circ} \mathrm{C}\right)$ [13]. Interestingly, after rewarming, d-ROM levels returned to control-group levels, different from the results reported in the present study, where MDA and carbonyl levels remained lower in the 


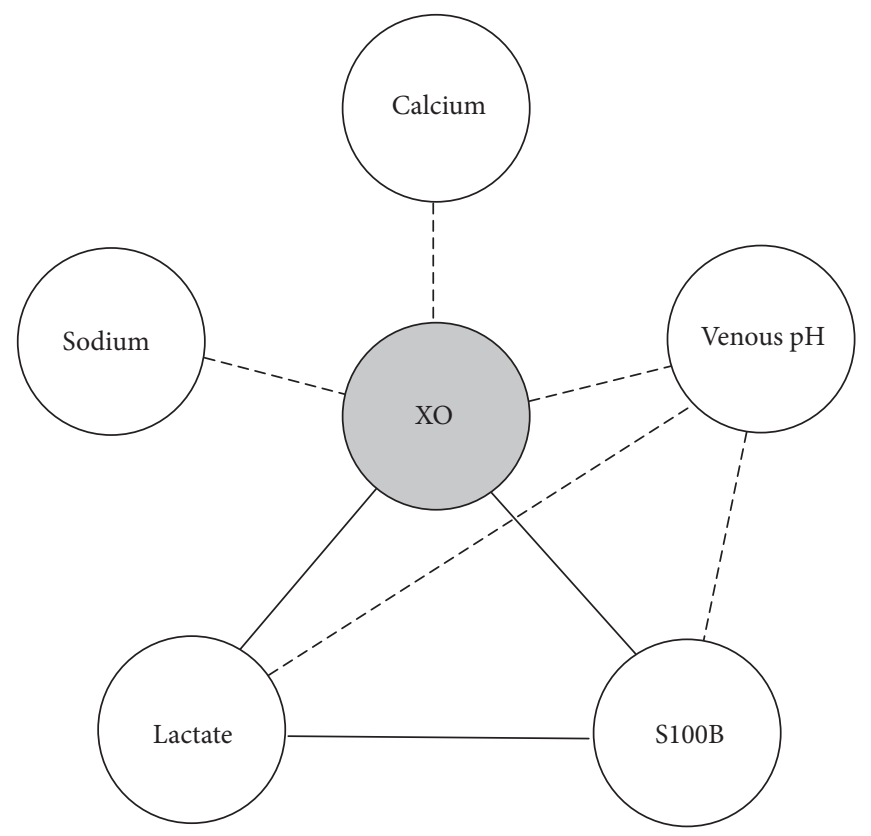

FIGURE 2: Schematic representation of the correlation analysis of xanthine oxidase (XO) activity. Lines indicate positive (solid lines) and negative (dotted lines) correlations.

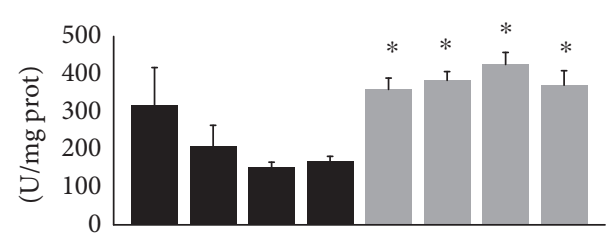

(a) SOD activity

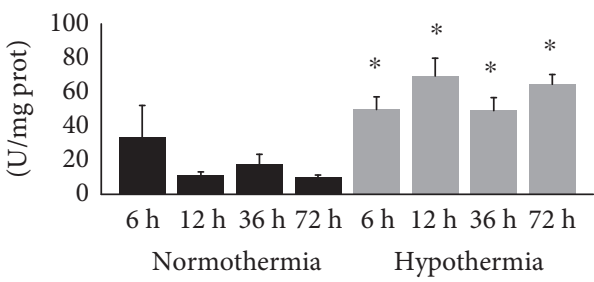

(c) GST activity

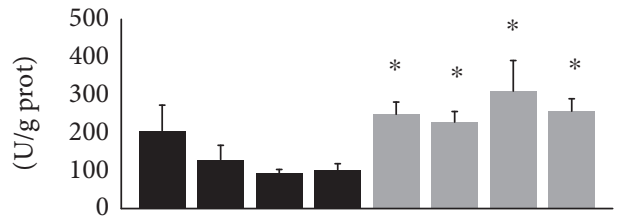

(b) GPx activity

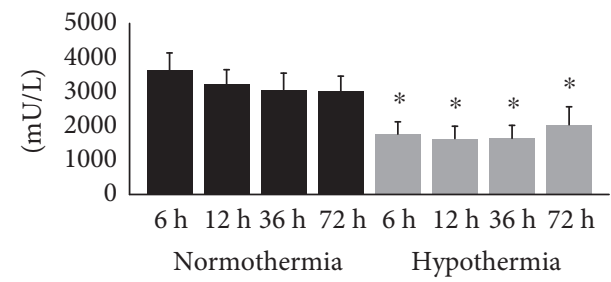

(d) PON1 activity

FIgURE 3: Antioxidant enzymatic activity at $6,12,36$, and $72 \mathrm{~h}$ after cardiac arrest (CA) in normothermic $(n=31)$ versus hypothermic $(n=11)$ patients. In hypothermic patients, body temperature reached $33^{\circ} \mathrm{C}$ at 6 and $12 \mathrm{~h}$ and $36^{\circ} \mathrm{C}$ at 36 and $72 \mathrm{~h}$ after CA. (a) Superoxide dismutase (SOD) activity; (b) glutathione peroxidase (GPx) activity; (c) glutathione S-transferase (GST) activity; (d) paraoxonase-1 (PON1) activity. Data are expressed as mean \pm standard error. ${ }^{*}$ Significantly different when comparing normothermic versus hypothermic groups at the same time point, $p \leq 0.05$.

hypothermic group even after rewarming. The difference between these results may indicate that, although high ROS generation returns after rewarming from $24 \mathrm{~h} \mathrm{MTH}$, protein damage and lipid peroxidation cascades may be impaired due to the activation of antioxidant defenses by MTH. The d-ROM test indicates the ability of a sample to generate peroxides, called "peroxidizability," and needs to be carefully analyzed due to possible overestimation of peroxide levels [25]. It is a different methodological approach compared to carbonyl and MDA levels, specific markers of the oxidation of amino acids residues and lipids, respectively.

In a porcine model of CA, MTH also reduced serum reactive oxygen metabolite levels compared to animals under controlled normothermia [12]. In I/R rat models, MDA levels were increased in the liver [26, 27], lung, ileum [27], and skeletal muscle, and these levels were restored by MTH [28]. To the best of our knowledge, no previous study has reported on protein carbonyl as a marker of oxidative damage after CA. Nevertheless, carbonyl levels showed a 
profile similar to that of MDA levels in the present study, since MTH significantly reduced the levels of both markers during PCAS. These results support the hypothesis that oxidative damage is reduced by MTH.

Despite the well-characterized increase in XO activity generating ROS during I/R injury [29], most studies are still trying to characterize the mechanisms of $\mathrm{XO}$ regulation, including the increase in XOR expression [2] and redox modifications due to $\mathrm{I} / \mathrm{R}$ injury $[6,30]$. In this respect, this is a pioneering study evaluating $\mathrm{XO}$ activity in post-CA patients who received MTH. No significant difference in $\mathrm{XO}$ activity was found during MTH at 6 and $12 \mathrm{~h}$ after CA. However, after rewarming (at 36 and $72 \mathrm{~h}$ after CA), XO activity was decreased in the hypothermic group. It is important to point out that decreased XO activity at 36 and $72 \mathrm{~h}$ in the hypothermic group was not significantly different from that in the normothermic group at the same time points. Further studies are needed to elucidate the effect of temperature on XOR regulation.

Hypoxic acidosis plays a relevant role in XOR regulation [31], but its exact mechanism of action has yet to be described. It is possible that ion imbalance participates in the upregulation of $\mathrm{XO}$ in $\mathrm{I} / \mathrm{R}$ injury together with the changes observed in oxygen saturation. During post-CA acidosis, blood calcium, sodium, and other electrolytes are increased as a compensatory mechanism for high lactate levels [7]. Interestingly, the present results show that XO activity was positively correlated with lactate and inversely correlated with $\mathrm{pH}$, calcium, and sodium, suggesting that $\mathrm{XO}$ regulation might have been related to acidosis in our patients. Although XO activity did not correlate with oxidative damage biomarkers in the present study, it was positively correlated with S100B levels. Despite our initial hypothesis that XO activity would correlate with MDA and carbonyl levels, it is possible that the different profiles found in $\mathrm{XO}$ activity as compared to the markedly low carbonyl and MDA levels in the hypothermic group may explain this lack of correlation.

4.2. Antioxidant Activity. In the present study, the activities of erythrocyte antioxidant enzymes SOD, GPx, and GST showed a similar marked increase after MTH during PCAS, as compared to the normothermic group, and were positively correlated with each other. The activity of antioxidant enzymes is altered as a consequence of I/R injury after $\mathrm{CA}$, and hypothermia may restore impaired defense mechanisms. Despite the paucity of studies evaluating antioxidant defenses in post-CA patients, there is some evidence from $\mathrm{I} / \mathrm{R}$ models. In rat models of $\mathrm{I} / \mathrm{R}$ injury, SOD activity was decreased in the liver, lung, and ileum [26-28]. A study in the liver found the same profile for SOD and hydrogen peroxide consumption, but GPx activity increased after I/R injury [26]. Similar to the present results, I/R injury of skeletal muscle in rats decreased the activity of SOD and GPx, and tissue activities were restored by MTH [28]. $\mathrm{MTH}$ also increased manganese SOD (MnSOD) expression in the frontal cortex of pigs after CA [11]. No studies were found relating GST, a detoxification enzyme, to CA or I/R injury.
MTH decreased the plasma biological antioxidant potential (BAP) during treatment in post-CA patients, returning to control levels after rewarming [13]. BAP was also evaluated in the serum of animals receiving $\mathrm{MTH}$ in a porcine model of CA, but no significant difference was found [12]. The BAP provides a global measurement of many enzymatic and nonenzymatic antioxidants together, indistinctly. Data from both studies do not support our results; however, instead of using the BAP test, we used specific methods for each antioxidant enzyme.

To the best of our knowledge, the present study is the first to evaluate PON1 activity in post-CA patients, and a significant decrease in PON1 activity was observed after MTH. PON1 activity was found to be reduced after I/R injury in the serum, liver, kidney, and lung of rats [32]. In patients undergoing coronary intervention with no-reflow, an I/R event, blood PON1 activity was lower after ischemia than that in patients with normal flow [33]. Interestingly, serum PON1 activity showed an inverse profile as compared to erythrocyte antioxidant enzymes SOD, GPx, and GST. Moreover, there was no correlation between serum PON1 activity and erythrocyte enzymes. These findings suggest that erythrocyte SOD, GPx, and GST are regulated differently from serum PON1 during I/R injury and MTH treatment.

\subsection{Neuronal Injury and Outcome Biomarkers. Controversy} has arisen over the efficacy of MTH following CA. After MTH was recommended by international resuscitation guidelines $[34,35]$, many studies began to point out that the reduction of neuronal damage by MTH is based on the control of body temperature rather than on hypothermia per se. When comparing treatments at a target temperature of $33^{\circ} \mathrm{C}$ (473 patients) and $36^{\circ} \mathrm{C}$ (466 patients), no difference was observed in neurological outcome [36]. However, other studies have pointed out the benefits of MTH by comparing hypothermic to controlled normothermic temperature management. In a porcine model of CA, by comparing controlled temperatures of $33^{\circ} \mathrm{C}$ and $36.8^{\circ} \mathrm{C}$, the $33^{\circ} \mathrm{C}$ group showed improved brain oxygen saturation and blood pressure, followed by a decrease in organ damage biomarkers in the heart, liver, and kidney. Interestingly, the levels of the marker of neuronal injury, neuron-specific enolase, were not altered by hypothermia [12]. Although S100B is considered a better marker of poor neurological outcome as compared to neuron-specific enolase [37], in the present study, S100B levels were not altered by MTH as well. Control and maintenance of body temperature below $37^{\circ} \mathrm{C}$ in the normothermic group may explain the similar rates of survival and $\mathrm{S} 100 \mathrm{~B}$ levels in hypothermic and normothermic patients.

Hs-CRP levels, widely used as marker of inflammation, were not altered by hypothermia. Its levels are inside the range expected for CA patients submitted to MTH [38]; however, no previous studies comparing hs-CRP levels in normothermia versus hypothermia patients were found.

Decreased glucose and elevated lactate levels are well known after CA due to anaerobic glycolysis [7]. Reduced blood glucose levels were found at 6 and $12 \mathrm{~h}$ after CA in the normothermic group, and these levels were increased by MTH. Although it is known that hypothermia increases 
glucose concentration, parsimony is required to evaluate glucose data in post-CA patients, since glucose levels are actively controlled in the ICU.

High lactate levels and impaired lactate clearance are frequently associated with poor outcome after CA [16, 39]. The decreased lactate levels observed after $12 \mathrm{~h}$ in the present study were expected due to lactate clearance from the circulation. Lactate levels play an important role in the metabolic acidosis consequent of CA, and the acidosis may be attenuated by increased calcium and sodium levels [7]. In the present study, $\mathrm{pH}$, lactate, calcium, and sodium were not altered by $\mathrm{MTH}$, suggesting hypothermia may not contribute to the attenuation of post-CA acidosis.

4.4. Limitations. The main limitation of this study is the lack of a control group of patients who did not undergo CA, making it difficult to discuss the influence of CA alone on oxidative stress parameters. In this respect, experimental models of CA allow a clearer view of PCAS. The study is also limited by the lack of pre-CA biochemical and clinical data, which could not be obtained due to the large number of out-of-hospital CA patients. Another limitation relates to differences in medication, since hypothermic patients received specific medication during MTH. Due to the high complexity of and time required for sample collection, the small number of patients, especially in the hypothermic group, is an important limitation. Finally, the lack of sample collection at a time point closer to the time of CA is also a limitation of the study; however, we determined $6 \mathrm{~h}$ after CA as the first sampling time point to avoid disturbing unstable patients and, more importantly, to avoid interrupting patient care.

\section{Conclusion}

In the present study, MTH reduced MDA and protein carbonyl levels as compared to controlled normothermia. An increase in the activity of erythrocyte antioxidant enzymes SOD, GPx, and GST occurred concomitantly with a decrease in the serum antioxidant activity of PON1 in patients treated with MTH. Moreover, hypothermia and acidosis may alter $\mathrm{XO}$ activity after CA. Altogether, the present results support our initial hypothesis by providing strong evidence that hypothermia can reduce oxidative stress in post-CA patients.
Abbreviations
BAP: Biological antioxidant potential
CA: Cardiac arrest
CPR: Cardiopulmonary resuscitation
GPx: Glutathione peroxidase
GST: Glutathione S-transferase
hs-CRP: High-sensitivity C-reactive protein
ICU: Intensive care unit
I/R: Ischemia-reperfusion
MDA: Malondialdehyde
MTH: Mild therapeutic hypothermia
$\mathrm{NAD}^{+}$: Nicotinamide adenine dinucleotide
PCAS: Postcardiac arrest syndrome
PON1: Paraoxonase-1

ROS: $\quad$ Reactive oxygen species

ROSC: Return of spontaneous circulation

S100B: S100 calcium-binding protein beta

SOD: Superoxide dismutase

XO: Xanthine oxidase

XOR: Xanthine oxidoreductase.

\section{Disclosure}

The authors alone are responsible for the content and writing of this paper.

\section{Conflicts of Interest}

The authors declare that there are no conflicts of interest.

\section{Authors' Contributions}

Fernanda S. Hackenhaar conducted the research, analyzed the data, and wrote the paper; Fernanda S. Hackenhaar, Tássia M. Medeiros, Fernanda M. Heemann, Camile S. Behling, Jordana S. Putti, Camila D. Mahl, Cleber Verona, and Ana Carolina A. da Silva contributed to the sample collection and experimental work; Maria C. Guerra and Carlos A. S. Gonçalves contributed to the experimental work and obtained the essential reagents and equipment; Vanessa Oliveira, Diego F. M. Riveiro, Silvia R. R. Vieira, and Mara S. Benfato designed and supervised the study.

\section{Acknowledgments}

This study was supported by grants from Fundo de Incentivo à Pesquisa do Hospital de Clinicas de Porto Alegre (FIPEHCPA), Coordenação de Aperfeiçoamento de Pessoal de Nível Superior (Capes), Fundação de Amparo e Pesquisa do Rio Grande do Sul (Fapergs), and Programa de PósGraduação em Biologia Celular e Molecular of Universidade Federal do Rio Grande do Sul (PPGBCM-UFRGS).

\section{References}

[1] J. P. Nolan, R. W. Neumar, C. Adrie et al., "Post-cardiac arrest syndrome: epidemiology, pathophysiology, treatment, and prognostication a scientific statement from the international liaison committee on resuscitation; the american heart association emergency cardiovascular care committee; the council on cardiovascular surgery and anesthesia; the council on cardiopulmonary, perioperative, and critical care; the council on clinical cardiology; the council on stroke," Resuscitation, vol. 79, no. 1, pp. 350-379, 2008.

[2] Y.-S. Zhang, B. Liu, X.-J. Luo et al., "A novel function of nuclear nonmuscle myosin regulatory light chain in promotion of xanthine oxidase transcription after myocardial ischemia/reperfusion," Free Radical Biology \& Medicine, vol. 83, pp. 115-128, 2015.

[3] F. S. Hackenhaar, F. Fumagalli, G. Li Volti et al., "Relationship between post-cardiac arrest myocardial oxidative stress and myocardial dysfunction in the rat," Journal of Biomedical Science, vol. 21, no. 1, p. 70, 2014.

[4] J. F. Gielis, G. A. Boulet, J. J. Briede et al., "Longitudinal quantification of radical bursts during pulmonary ischaemia 
and reperfusion," European Journal of Cardio-Thoracic Surgery, vol. 48, no. 4, pp. 622-628, 2015.

[5] M. G. Angelos, V. K. Kutala, C. A. Torres et al., "Hypoxic reperfusion of the ischemic heart and oxygen radical generation," American Journal of Physiology. Heart and Circulatory Physiology, vol. 290, no. 1, pp. 341-347, 2006.

[6] M. G. Battelli, A. Bolognesi, and L. Polito, "Pathophysiology of circulating xanthine oxidoreductase: new emerging roles for a multi-tasking enzyme," Biochimica et Biophysica Acta, vol. 2014, no. 9, pp. 1502-1517, 1842.

[7] J. Makino, S. Uchino, H. Morimatsu, and R. Bellomo, "A quantitative analysis of the acidosis of cardiac arrest: a prospective observational study," Critical Care, vol. 9, no. 4, pp. 357-362, 2005.

[8] J.-C. Orban, A. Scarlatti, P.-E. Danin, J. Dellamonica, G. Bernardin, and C. Ichai, "Assessment of muscle tissue oxygen saturation after out-of-hospital cardiac arrest," Journal of Critical Care, vol. 30, no. 6, pp. 1184-1189, 2015.

[9] Y. Li, G. Ristagno, J. Guan et al., "Preserved heart rate variability during therapeutic hypothermia correlated to 96 hrs neurological outcomes and survival in a pig model of cardiac arrest," Critical Care Medicine, vol. 40, no. 2, pp. 580-586, 2012.

[10] C. Testori, F. Sterz, W. Behringer et al., "Mild therapeutic hypothermia is associated with favourable outcome in patients after cardiac arrest with non-shockable rhythms," Resuscitation, vol. 82, no. 9, pp. 1162-1167, 2011.

[11] P. Gong, C.-S. Li, R. Hua et al., "Mild hypothermia attenuates mitochondrial oxidative stress by protecting respiratory enzymes and upregulating MnSOD in a pig model of cardiac arrest," PLoS One, vol. 7, no. 4, article e35313, 2012.

[12] P. Ostadal, M. Mlcek, A. Kruger et al., "Mild therapeutic hypothermia is superior to controlled normothermia for the maintenance of blood pressure and cerebral oxygenation, prevention of organ damage and suppression of oxidative stress after cardiac arrest in a porcine model," Journal of Translational Medicine, vol. 11, no. 1, p. 124, 2013.

[13] K. Dohi, K. Miyamoto, K. Fukuda et al., "Status of systemic oxidative stress during therapeutic hypothermia in patients with post-cardiac arrest syndrome," Oxidative Medicine and Cellular Longevity, vol. 2013, Article ID 562429, p. 8, 2013.

[14] R. Starodub, B. S. Abella, A. V. Grossestreuer et al., "Association of serum lactate and survival outcomes in patients undergoing therapeutic hypothermia after cardiac arrest," Resuscitation, vol. 84, no. 8, pp. 1078-1082, 2013.

[15] K. Leon, K. Pichavant-Rafini, H. Ollivier, and E. L'Her, "Effect of induced mild hypothermia on acid-base balance during experimental acute sepsis in rats," Therapeutic Hypothermia and Temperature Management, vol. 5, no. 3, pp. 163-170, 2015.

[16] A. Zeiner, M. Holzer, F. Sterz et al., "Hyperthermia after cardiac arrest is associated with an unfavorable neurologic outcome," Archives of Internal Medicine, vol. 161, no. 6, pp. 2007-2012, 2001.

[17] M. C. Leite, F. Galland, G. Brolese et al., "A simple, sensitive and widely applicable ELISA for S100B: methodological features of the measurement of this glial protein," Journal of Neuroscience Methods, vol. 169, no. 1, pp. 93-99, 2008.

[18] R. L. Levine, "Carbonyl modified proteins in cellular regulation, aging, and disease," Free Radical Biology \& Medicine, vol. 32, no. 9, pp. 790-796, 2002.
[19] M. Karatepe, "Simultaneous determination of ascorbic acid and free malondialdehyde in human serum by HPLC-UV," Lc Gc North America, vol. 22, no. 4, pp. 104-106, 2004.

[20] S. Tsuchida, Experimental Protocols for Reactive Oxygen and Nitrogens Species, pp. 83-85, London, Oxford University Press, 2000.

[21] T. M. van Himbergen, M. Roest, J. de Graaf et al., "Indications that paraoxonase-1 contributes to plasma high density lipoprotein levels in familial hypercholesterolemia," Journal of Lipid Research, vol. 46, no. 3, pp. 445-451, 2005.

[22] H. W. Eckerson, C. M. Wyte, and B. N. Ladu, "The humanserum paraoxonase arylesterase polymorphism," American Journal of Human Genetics, vol. 35, no. 6, pp. 1126-1138, 1983.

[23] M. M. Bradford, "Rapid and sensitive method for quantitation of microgram quantities of protein utilizing principle of protein-dye binding," Analytical Biochemistry, vol. 72, no. 1-2, pp. 248-254, 1976.

[24] S. Turedi, A. Gunduz, A. Mentese et al., "Investigation of the possibility of using ischemia-modified albumin as a novel and early prognostic marker in cardiac arrest patients after cardiopulmonary resuscitation," Resuscitation, vol. 80, no. 9, pp. 994-999, 2009.

[25] B. Halliwell and J. M. C. Gutteridge, Free Radicals in Biology and Medicine, Oxford University Press, Oxford, 2015.

[26] S. A. Abdel-Gaber, M. A. Ibrahim, E. F. Amin, S. A. Ibrahim, R. K. Mohammed, and A. M. Abdelrahman, "Effect of selective versus non-selective cyclooxygenase inhibitors on ischemiareperfusion-induced hepatic injury in rats," Life Sciences, vol. 134, pp. 42-48, 2015.

[27] I. O. Tekin, E. Y. Sipahi, M. Comert, S. Acikgoz, and G. Yurdakan, "Low-density lipoproteins oxidized after intestinal ischemia/reperfusion in rats," The Journal of Surgical Research, vol. 157, no. 1, pp. 47-54, 2009.

[28] H. Ozkan, S. Ekinci, B. Uysal et al., "Evaluation and comparison of the effect of hypothermia and ozone on ischemiareperfusion injury of skeletal muscle in rats," The Journal of Surgical Research, vol. 196, no. 2, pp. 313-319, 2015.

[29] J. L. Zweier, P. Kuppusamy, and G. A. Lutty, "Measurement of endothelial-cell free-radical generation - evidence for a central mechanism of free-radical injury in postischemic tissues," Proceedings of the National Academy of Sciences of the United States of America, vol. 85, no. 11, pp. 4046-4050, 1988.

[30] Y. Zhang, S. Hu, and Y. Chen, "Hepatocyte growth factor suppresses hypoxia/reoxygenation-induced xo activation in cardiac microvascular endothelial cells," Heart and Vessels, vol. 30, no. 4, pp. 534-544, 2015.

[31] R. S. Khambata, S. M. Ghosh, and A. Ahluwalia, "“'Repurposing" of xanthine oxidoreductase as a nitrite reductase: a new paradigm for therapeutic targeting in hypertension," Antioxidants \& Redox Signaling, vol. 23, no. 4, pp. 340-353, 2015.

[32] A. Tufek, O. Tokgoz, I. Aliosmanoglu et al., "The protective effects of dexmedetomidine on the liver and remote organs against hepatic ischemia reperfusion injury in rats," International Journal of Surgery, vol. 11, no. 1, pp. 96-100, 2013.

[33] M. Gur, C. Turkoglu, A. Taskin et al., "Paraoxonase-1 activity and oxidative stress in patients with anterior ST elevation myocardial infarction undergoing primary percutaneous coronary intervention with and without no-reflow," Atherosclerosis, vol. 234, no. 2, pp. 415-420, 2014.

[34] M. Holzer, E. Cerchiari, P. Martens, and Hypothermia after Cardiac Arrest Study Group, "Mild therapeutic hypothermia 
to improve the neurologic outcome after cardiac arrest," The New England Journal of Medicine, vol. 346, no. 8, p. 1756, 2002.

[35] S. A. Bernard, T. W. Gray, M. D. Buist et al., "Treatment of comatose survivors of out-of-hospital cardiac arrest with induced hypothermia," The New England Journal of Medicine, vol. 346, no. 8, pp. 557-563, 2002.

[36] N. Nielsen, J. Wettersley, T. Cronberg et al., "Targeted temperature management at 33 degrees $\mathrm{C}$ versus 36 degrees $\mathrm{C}$ after cardiac arrest," The New England Journal of Medicine, vol. 369, no. 23, pp. 2197-2206, 2013.

[37] K. Shinozaki, S. Oda, T. Sadahiro et al., "Serum S-100B is superior to neuron-specific enolase as an early prognostic biomarker for neurological outcome following cardiopulmonary resuscitation," Resuscitation, vol. 80, no. 8, pp. 870-875, 2009.

[38] A. M. Dell'anna, J. B. Viotti, M. Beumier et al., "C-reactive protein levels after cardiac arrest in patients treated with therapeutic hypothermia," Resuscitation, vol. 108, no. 7, p. $111,2016$.

[39] M. W. Donnino, J. Miller, N. Goyal et al., "Effective lactate clearance is associated with improved outcome in postcardiac arrest patients," Resuscitation, vol. 75, no. 2, pp. 229234, 2007. 


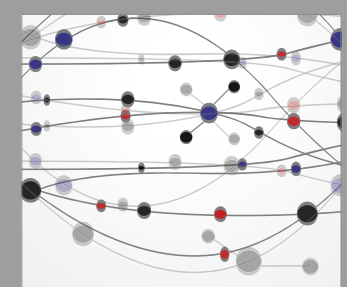

The Scientific World Journal
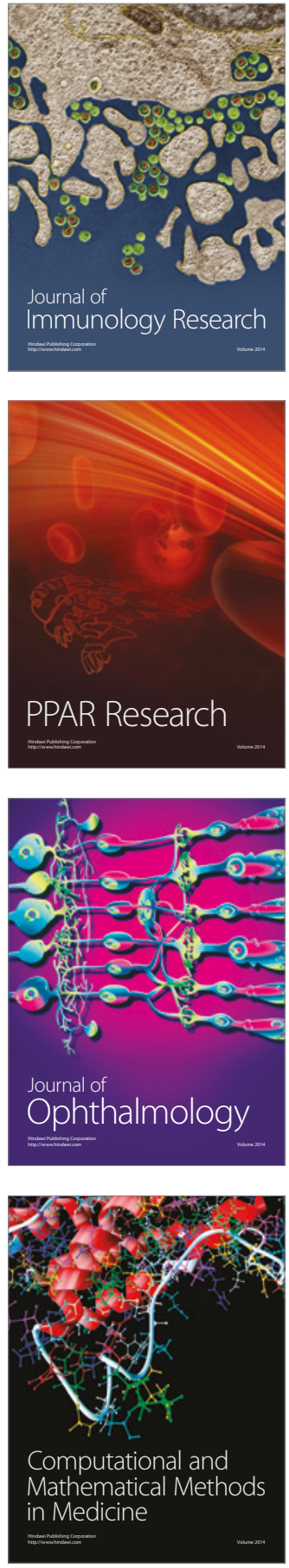

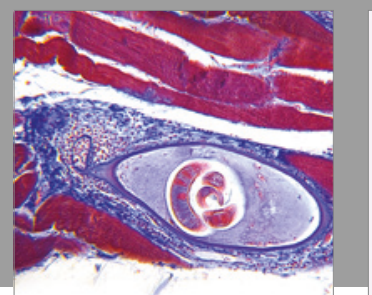

Gastroenterology Research and Practice
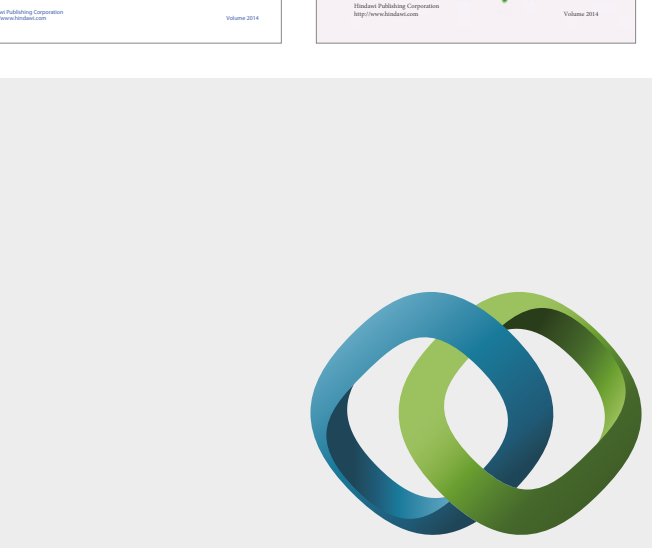

\section{Hindawi}

Submit your manuscripts at

https://www.hindawi.com
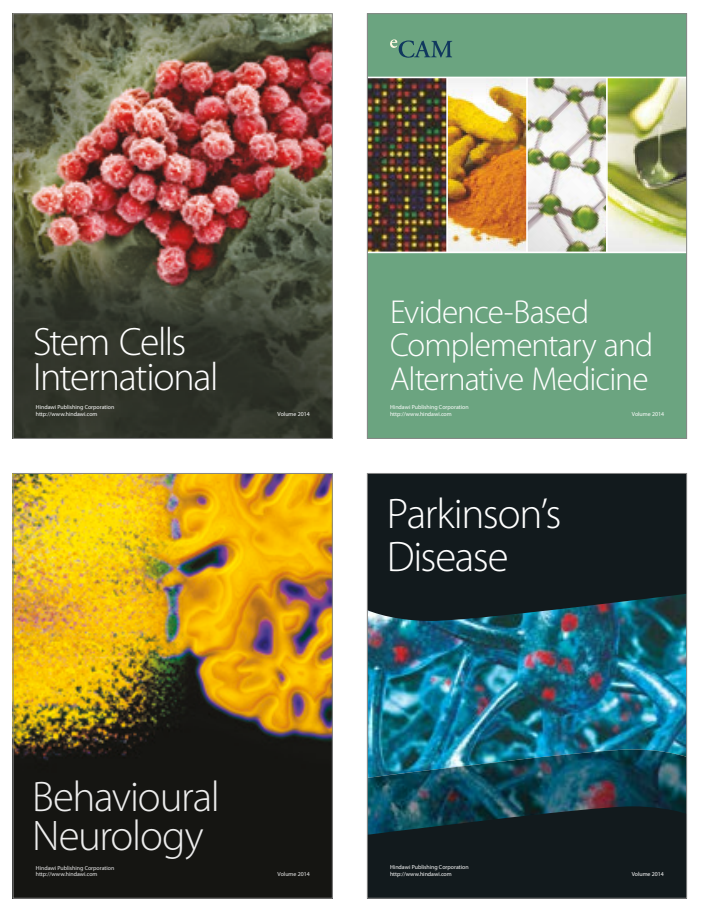
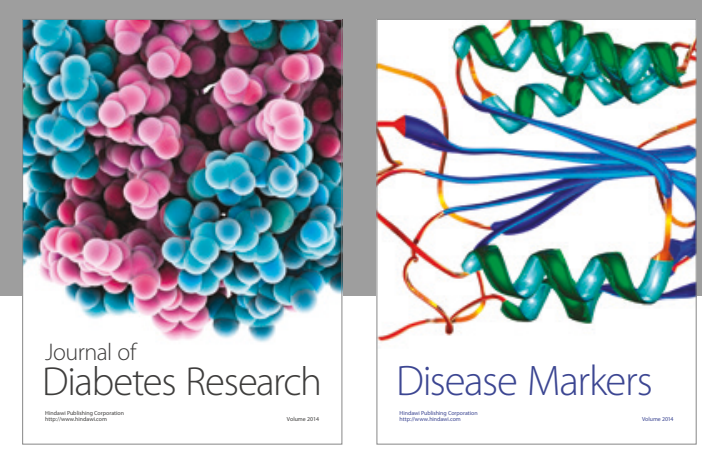

Disease Markers
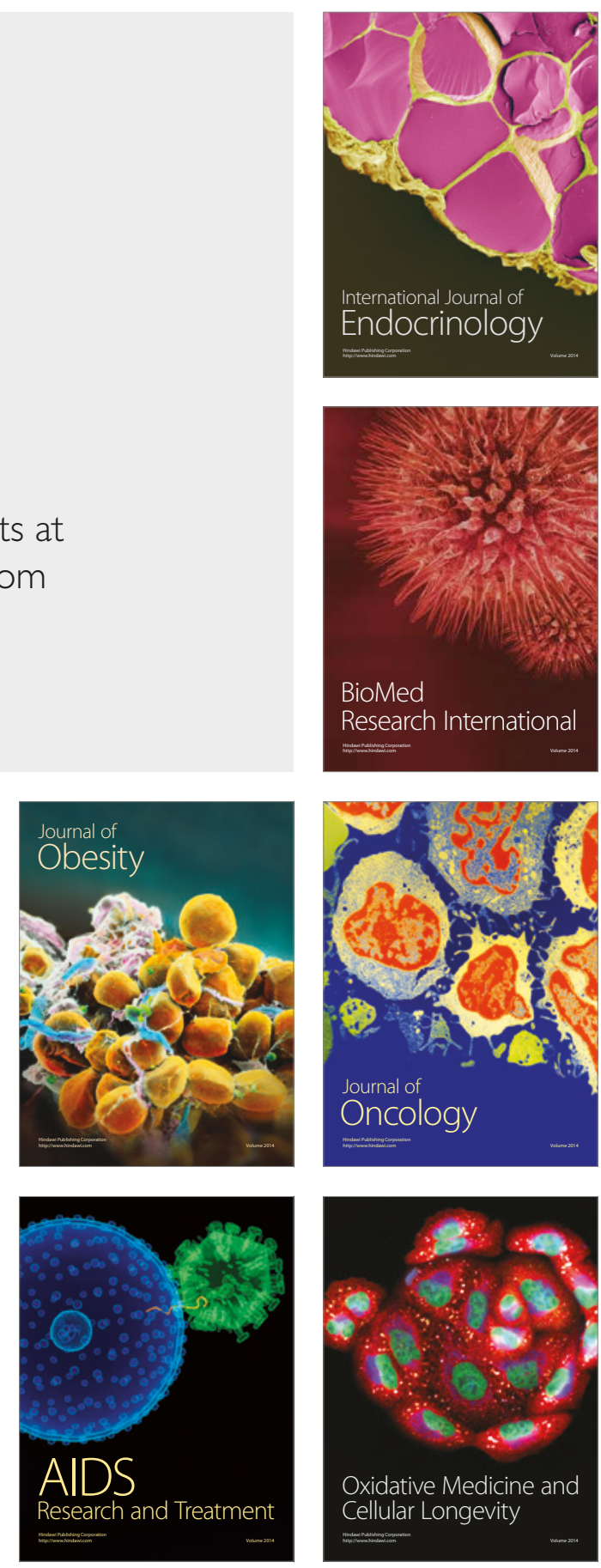